EXTENDED REPORT

\title{
Carboxymethylated chitin reduces MMP-1 expression in rabbit ACLT osteoarthritic cartilage
}

\author{
W Hongbin, D Jingyuan, C Linyun, D Yuming
}

\begin{abstract}
See end of article for authors' affiliations

....................

Correspondence to: Dr H Wu, Orthopaedic Department of Union Hospital, Tongji Medical College of Huazhong Science and Technology University, Wuhan 430022, People's Republic of China; 13971454014@ vip.sina.com
\end{abstract}

Accepted 13 May 2003

\begin{abstract}
Objective: To examine the in vivo effects of carboxymethylated chitin (CMC), intra-articularly administered, on cartilage degradation and the level and distribution of cartilage matrix metalloproteinase-1 (MMP-1).

Methods: Osteoarthritis (OA) was induced in 20 rabbits by unilateral anterior cruciate ligament transection (ACLT). The experimental group, comprising 10 rabbits randomly selected, was given an intraarticular injection of $0.3 \mathrm{ml}$ of $2 \%$ CMC solution at 1, 3, and 5 weeks after ACLT. A further 10 rabbits that received an intra-articular injection of $0.3 \mathrm{ml}$ normal saline at the same time served as controls. All knees were harvested at 6 weeks after surgery. Cartilage degradation of femoral condyles was evaluated at two levels: macroscopic and light microscopic. Tissue level and distribution of MMP-1 was documented by reverse transcriptase-polymerase chain reaction (RT-PCR) and immunohistochemistry.

Results: Cartilage degradation in the control group was significantly more severe than that in the experimental group both on the macroscopic grading scale and on Mankin's grading scale. In RT-PCR the amount of MMP-1 was significantly reduced by the treatment of CMC. Immunohistochemical study showed that in the experimental group MMP-1 was predominantly expressed in the superficial and upper intermediate layers of cartilage, and the amount of MMP-1 in the experimental group was also lower than that in control group.

Conclusion: CMC significantly reduces the severity of cartilage degradation and reduces the expression of MMP-1 in cartilage, both at the mRNA and the protein level, and thus may be a potential drug for the treatment of $\mathrm{OA}$.
\end{abstract}

$\mathrm{O}$ steoarthritis (OA) is characterised by chronic and progressive cartilage destruction, resulting in recurrent pain and gradual loss of articular function. Although the initial causes of $\mathrm{OA}$ are still obscure, the mechanism of cartilage degradation has been extensively investigated, and it is now generally agreed that cartilage degradation is a process resulting from an imbalance of anabolic and catabolic changes in the articular cartilage matrix. The process involves many biochemical mediators such as interleukin 1, nitric oxide (NO), and matrix metalloproteinases (MMPs). Among these mediators, MMPs can directly destroy nearly all components of the cartilage matrix, and they have a major impact on OA degeneration. ${ }^{12}$ Recently, a new avenue of research has focused on finding agents aimed at reducing the catabolic changes in OA, thus slowing or reversing OA progression. It is believed that many bioagents based on glycosaminoglycans (GAGs) have chondroprotective effects..$^{3-5}$ Chitinous materials are the most abundant materials rich in GAGs known. Because of their good biocompatibility and biodegradability, they have been investigated and used for a long time in many medical fields, such as artificial skin, prevention of after surgery adhesion, and as a drug vehicle. Recently, these materials have also been extensively studied in cartilage tissue engineering to examine their potential as carriers of chondrocyte and related growth factors. ${ }^{67}$ In view of their potential to repair cartilage, we prepared a new chitin derivative: carboxymethylated chitin (CMC), which has greatly increased solubility, and observed the effects of intra-articular injection of its solution on cartilage degradation and the expression of MMP-1 in experimentally induced rabbit anterior cruciate ligament transection (ACLT) OA.

\section{MATERIALS AND METHODS}

\section{Animals}

Twenty white rabbits weighing about $2.5-3 \mathrm{~kg}$ were bought from the animal centre of Tongji Medical College of Huazhong Science and Technology University. All rabbits were anaesthetised by intramuscular injection of ketomine $(100 \mathrm{mg} / \mathrm{kg}$ ) and received unilateral ACLT. During the first 4 days after the operation each rabbit received an intramuscular injection of 400000 units of penicillin every day to prevent infection. After ACLT, each rabbit was placed in a cage $(60 \mathrm{~cm} \times 60 \mathrm{~cm} \times 40 \mathrm{~cm})$ without any further immobilisation. Ten randomly selected rabbits formed the experimental group and were given an intra-articular injection of $0.3 \mathrm{ml} 2 \%$ CMC solution at 1,3 , and 5 weeks after surgery. The other 10 rabbits comprised the control group and received an intra-articular injection of $0.3 \mathrm{ml}$ normal saline at the same time.

\section{Macroscopic morphology}

Knees were harvested at 6 weeks after ACLT. Degenerative changes of femoral condyles in the cartilage were observed under dissecting microscope and the OA severity was graded on a scale of 0-4 as follows: $0=$ surface smooth with normal colour; $1=$ surface rough with minimal fibrillation or a slight yellowish discolouration; 2 = cartilage erosion extending into the superficial or middle layers; 3 =cartilage ulceration

Abbreviations: $A C L T$, anterior cruciate ligament transection; $C M C$, carboxymethylated chitin; GAG, glycosaminoglycan; GAPDH, glyceraldehyde-3-phosphate dehydrogenase; MMP, matrix metalloproteinase; NO, nitric oxide; OA, osteoarthritis; OD, optical density; RT-PCR, reverse transcriptase-polymerase chain reaction 
extending into the deep layers; $4=$ cartilage depletion with subchondral bone exposed. ${ }^{8}$

\section{Light microscopic histology}

The medial sides of the medial femoral condyles were cut along the sagittal plane, and $3 \mathrm{~mm}$ thick cartilage blocks with subchondral bone were obtained. These were then fixed in $4 \%$ paraformaldehyde at $4^{\circ} \mathrm{C}$ for 24 hours and decalcified in $15 \%$ EDTA for 4 weeks. All samples were embedded in paraffin. Serial sections $(6 \mu \mathrm{m})$ were cut and stained with haematoxylin and eosin and safranin O-fast green. The latter staining was carried out under the following conditions: dye concentration $0.1 \%$, staining time 6 minutes. The severity of OA was graded on a scale of $0-14$ by two independent observers, using Mankin's histological/histochemical scale. The final score of each sample was the average of two independent scores. ${ }^{910}$

\section{Reverse transcriptase-polymerase chain studies}

Cartilage samples from the degenerative region of the medial femoral condyle were collected. They were ground and homogenised in $1 \mathrm{ml}$ TRIzol reagent. Total RNA was extracted according to the manufacturer's instructions; the RNA concentration was determined by measuring the optical density at $260 \mathrm{~nm}\left(\mathrm{OD}_{260}\right)$. The $\mathrm{OD}_{260} / \mathrm{OD}_{280}$ ratios were $>1.8$. Total RNA ( $1 \mu \mathrm{g}$ ) was reverse transcribed into cDNA as follows: $12.5 \mu \mathrm{l}$ reaction mixture containing $1 \mu \mathrm{g}$ of total RNA, $1 \mu \mathrm{l}$ oligo $\mathrm{d}(\mathrm{t})$, and water distilled three times was denatured at $70^{\circ} \mathrm{C}$ for 5 minutes, then immediately transferred to an ice box. After adding $0.5 \mu \mathrm{l}$ RNAs in $2 \mu \mathrm{l} 10 \mathrm{mM}$ dNTP, $4 \mu \mathrm{l} 5 \times$ buffer, and $1 \mu \mathrm{l}$ RTase, the mixture was allowed to react at $37^{\circ} \mathrm{C}$ for 60 minutes, then at $95^{\circ} \mathrm{C}$ for 5 minutes. Amplification of the generated cDNA was performed in a final volume of $50 \mu \mathrm{l}$ reaction mixture containing $34.7 \mu \mathrm{l}$ water distilled three times, $1 \mu \mathrm{l} 10 \mathrm{mM}$ dNTP, $4 \mu \mathrm{l} 25 \mathrm{mM} \mathrm{MgCl}_{2}, 5 \mu \mathrm{l} 10 \times$ buffer, $0.4 \mu \mathrm{l}$ sense primer, $0.4 \mu \mathrm{l}$ antisense primer, $4 \mu \mathrm{l}$ cDNA, and $0.5 \mu \mathrm{l}$ Taq enzyme. Thirty cycles of amplification were performed for glyceraldehyde-3-phosphate dehydrogenase (GAPDH) and 35 cycles for MMP-1 following the procedure recommended by Perkin-Elmer: 120 seconds at $95^{\circ} \mathrm{C}, 30$ seconds at $60^{\circ} \mathrm{C}$, 90 seconds at $72^{\circ} \mathrm{C}$. The oligonuleotide primer pairs used for GAPDH and MMP-l were as follows: GAPDH (sense: TGAACGGATTTGGCCGCATT, antisense: ATTCACGCCCATC ACAAACA), MMP-1 (sense: AGAGCAAGATGTGGGATGG, antisense: CTTGACAGGTCTGGTGTGTA). ${ }^{11}$ Transcripts were analysed by $1.5 \%$ agarose gel electrophoresis and visualised by ethidium bromide staining. After photographing the gels under ultraviolet light, the intensity of the bands corresponding to MMP-1 was quantified by densitometry scanning performed with the Champion Gel Image Analysis System and normalised to GAPDH mRNA levels.

\section{Immunohistochemical studies for MMP-1}

Serial $6 \mu \mathrm{m}$ sections were floated onto poly-L-lysine coated slides, deparaffinised in toluene, hydrated in a graded series of ethanol solutions, and preincubated with $0.5 \mathrm{mg} / \mathrm{ml}$ bovine testicular hyaluronidase (Sigma) at $37^{\circ} \mathrm{C}$ for $40 \mathrm{~min}$ utes, then incubated in $0.3 \%$ hydrogen peroxide/methanol for 15 minutes. Slides were further incubated with $2 \%$ normal bovine serum for 20 minutes, blotted, and then overlaid with a sheep antirabbit MMP-1 serum (a generous gift from Dr Nagase H, Medical Center, University of Kansas, USA) at a dilution of $1: 200$. They were incubated at $4{ }^{\circ} \mathrm{C}$ for 24 hours. A second antibody reaction was carried out for 20 minutes using biotinylated antisheep IgG antibody (Zhongshan Biotech, Beijing), followed by reaction with streptavidinperoxidase solution (Zhongshan Biotech, Beijing) for 20 minutes. All incubations were carried out in a humidified chamber and the colour developed with 3,3'-diaminobenzidine containing hydroxide peroxide. To determine the specificity of staining, two control procedures were employed according to the same experimental protocol: $(a)$ omission of the primary antibody; $(b)$ substitution of the primary antibody with non-immune goat serum.

\section{Morphometric analysis}

The presence of MMP-1 was estimated by determining the number of chondrocytes staining positive within the different layers of cartilage. Each cartilage section was divided into six high power microscopic fields ( $\times 400$ magnification). Three fields were situated at each of the following two zones: the superficial and upper intermediate layers, and the lower intermediate and deeper layers. For each arthritic specimen, it was ensured before evaluation that an intact cartilage surface could be detected and used as a marker for validation of morphometric analysis. The total number of chondrocytes and the number of chondrocytes staining positive for MMP-1 were estimated for the full thickness cartilage. The final results were expressed as the percentage of chondrocytes staining positive, with the maximum score being $100 \%{ }^{10}$ Each slide was evaluated under "blinded" conditions by two independent observers; the variation between the observers' findings was $<5 \%$.

\section{Statistical analysis}

Statistical analysis of macroscopic OA scores was performed using the Wilcoxon rank sum test. Other data were analysed using the $t$ test.

\section{RESULTS}

\section{Gross morphology}

The principal degenerative changes in the experimental group were cartilage roughness and erosion, all located on the medial side of the medial femoral condyles. Fibrocartilage proliferation on the medial edge of the medial femoral condyle was evident in three knees. Cartilage lesions in the control group were also located at the medial side of the medial femoral condyles, but the principal OA changes in the controls were cartilage ulceration, and the fibrocartilage proliferation became more evident. Table 1 shows the OA scores under dissecting microscope 6 weeks after ACLT, indicating that OA severity in the experimental group was significantly lower than that in the control group $(p<0.01)$.

\section{Light microscopic histology}

Diffuse hypercellularity or obvious chondrocyte clustering in the superficial and upper intermediate zones was frequently seen in many experimental cartilage samples. Some samples had cartilage fissures or clefts to the transitional zone; the tidemark was also disordered. Safranin O staining was slightly or moderately reduced. In the control groups we could see many erosions or ulceration extending into the radial or calcified zone of the cartilage, the number of chondrocytes was greatly decreased, and there were many

Table 1 OA scores under dissecting microscope at 6 weeks after ACLT $(p<0.01)$

\begin{tabular}{lll}
\hline Group score & Control group* & Experimental group* \\
\hline 0 & 0 & 0 \\
1 & 1 & 6 \\
2 & 4 & 4 \\
3 & 4 & 0 \\
4 & 1 & 0 \\
\hline \multirow{2}{*}{ The number of rabbits with the respective group score is } \\
given.
\end{tabular}


dead or collapsed cells. The tidemark was disarranged or had disappeared, and safranin O staining was severely reduced or completely lost. Mankin's score (mean (SEM)) for the medial femoral condyles in the experimental group was 6.2 (1.54), and for the control group 9.05 (1.95). Statistical analysis showed a significant difference between the two groups, indicating that the cartilage OA change in the experimental group was less than that in control group $(\mathrm{p}<0.01)$.

\section{RT-PCR analysis}

Amplification of mRNA yielded distinct bands of the expected length for MMP- 1 in all cartilage samples. Figure 1 showed the representative MMP-1 bands from the experimental and control groups. Densitometry showed that the intensity of band corresponding to MMP-1 in the cartilage of experimental group was $0.127(0.061)$, significantly lower than that of the control group, 0.259 (0.047), indicating that the amount of MMP-1 mRNA in the experimental group was significantly lower than that in the control group $(\mathrm{p}<0.001)$.

\section{Immunohistomorphometric analysis}

The pattern of MMP-l staining was diffuse dark brown colouration of the cytoplasm, which could be seen in the full thickness of the cartilage, but predominantly in chondrocytes from the superficial and upper intermediate layers. The mean (SEM) cell score in the experimental group was $21.6(9.7)$, significantly lower than that in the control group, 45.8 (17.4), indicating that the amount of MMP-1 protein in the experimental group was significantly lower than that in the control group $(\mathrm{p}<0.001)$ (figs $2 \mathrm{~A}$ and $\mathrm{B}$ ). Immunohistochemical controls using non-immune goat serum showed only background staining (fig 2C).

\section{DISCUSSION}

Recently, more and more investigations have indicated that bio-products made from natural materials rich in GAG have many chondroprotective effects, including improvement of cartilage matrix metabolism and help in cartilage repairing. Now many bio-products have been developed and successfully used in the treatment of OA, such as, glucosamine from the exoskeleton of crustaceans; Rumalon extracted from bovine cartilage and bone marrow and whose major constituents are GAG and several polypeptides; and hyaluronan extracted from rooster comb. ${ }^{3-5}$ Chitinous materials are the most abundant materials rich in GAGs known. Currently, more and more studies are underway to explore their potential in repairing cartilage tissue. Sechriest found that bovine primary articular chondrocytes, when seeded onto a thin layer of chondroitin sulphate A-chitosan-formed discrete, focal adhesions to the material and maintained many characteristics of the differentiated chondrocytic

GAPDH

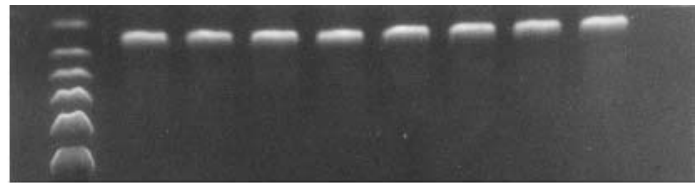

MMP-1

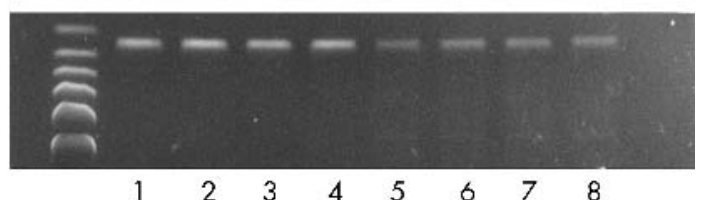

Figure 1 Representative bands of MMP-1 in RT-PCR electrophoresis (lanes 1-4, control group; lanes 5-8, experimental group): indicating that the expression of MMP-1 in the experimental group is significantly lower than that in control group. GAPDH is a reference gene; the lengths of the MMP-1 and GAPDH bands are $307 \mathrm{bp}$ and $302 \mathrm{bp}$, respectively. phenotype, including round morphology, collagen type II, and proteoglycan production. ${ }^{6}$ Lu et al studied the effects of intra-articular injection of chitosan solution on rat knee cartilages and found that chitosan significantly slowed the decrease in epiphysial cartilage thicknesses and significantly increased articular cartilage chondrocyte densities. ${ }^{12}$ Hwang found that many chitinous materials could greatly reduce the ability of macrophages to produce NO, while the latter is thought to be the most important inflammatory mediator in the development of OA, because it can induce the apoptosis of chondrocyte and increase the production and activity of MMPs. ${ }^{13}{ }^{14}$ All the above studies demonstrate that chitinous materials may be able to provide scaffold for the growth of chondrocytes, enhance cartilage repairing, and curb the inflammation of cartilage and synovium, thus might be useful for the treatment of OA. So we examined the effect of a newly prepared chitin derivative- $\mathrm{CMC}-$ on OA cartilage degradation, and we found that CMC could significantly reduce the severity of cartilage degradation in rabbit ACLT OA, with both macroscopic grading scores and Mankin's scores of the experimental knees being significantly lower than those of control knees $(\mathrm{p}<0.01)$. Compared with common chitin, CMC has greatly increased solubility, and $2 \%$ CMC in normal saline solution is transparent, colourless and viscous, and homogeneous, with a $\mathrm{pH}$ value 7.2. Furthermore, this solution has many characteristics similar to those of normal joint fluid and, theoretically, it can also supplement and increase the viscosity and elasticity of joint fluid, just like hyaluronan, when injected intra-articularly. Another advantage of CMC is that it shares more physical and chemical similarities with extracellular matrix polysaccharide than do common chitinous materials. ${ }^{15}$

The ACLT model has proved to be a useful tool in the investigation of OA development, it has biochemical and pathological changes identical to those found in human OA, and the lesion site is reproduced well in the medial femoral condyle, enabling good comparison before and after an intervention. It also permits a more in-depth analysis of the role of proteolytic enzymes in OA progression, which is hard to achieve using human OA specimens. ${ }^{16}$

Overexpression of proteolytic enzymes such as MMPs is believed to play an important part in the degradation and loss of cartilage matrix, which is an important process in OA. Because MMP-1 can directly cleave the chains of triple helical type II collagen, which is the predominant collagen in articular cartilage and the key to maintaining the integrity of cartilage, it has been thought to be an important participant in cartilage turnover. Now many investigations have also found that the level of MMP-1 is increased in the synovium, synovial fluid, and cartilage samples from humans with OA, suggesting that MMP-1 has a major role in the pathological loss of articular cartilage. ${ }^{17}{ }^{18}$ In this study we first demonstrate that CMC can reduce the expression of cartilage MMP-1. The mRNA level of MMP-1 detected by RTPCR in experimental cartilage was lower than that in control cartilage, and morphometric analysis also showed that the protein level in experimental cartilage was reduced compared with the controls. Of note, the MMP-1 positive chondrocytes were predominantly distributed in superficial and upper intermediate layers of cartilage, coinciding with the fact that in the early stage of $\mathrm{OA}$, disorganisation of the collagen network first occurs in the upper half of cartilage. ${ }^{19}$ This result showed again the important role of MMP-1 in the pathogenesis of OA, and also showed that the inhibition of MMP-1 by CMC could contribute to its ability to slow down the pathological changes of OA cartilage.

The mechanism behind the inhibition of CMCs on cartilage MMP-1 calls for further investigation. However, the following are likely candidates: 

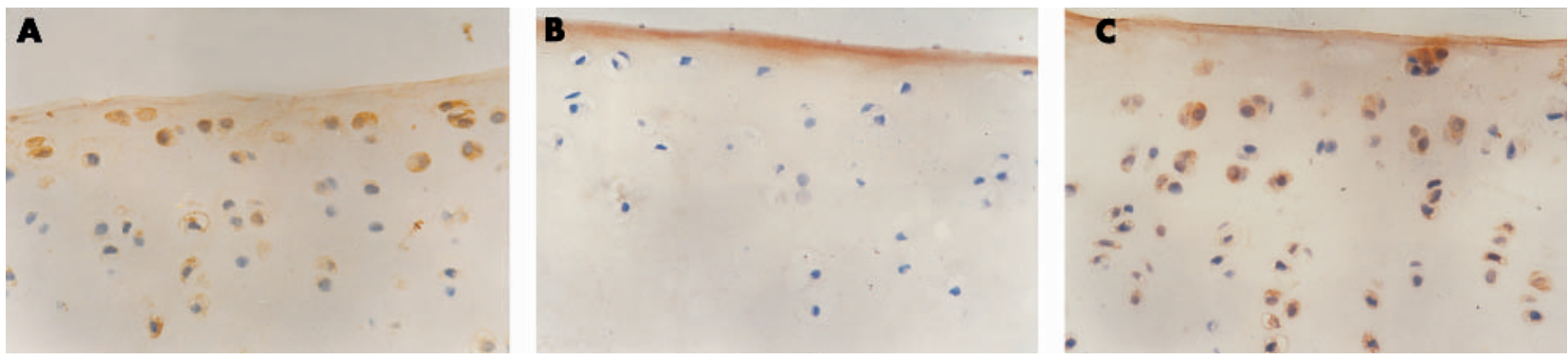

Figure 2 Immunohistochemistry study of MMP-1 (photomicrograph $\times 400$ ). (A) The experimental group, showing that MMP-1 positive cells are predominantly located in the superficial and upper intermediate layers. (B) The immunohistochemical control, showing only background staining (C) The control group, showing that the number of MMP-1 positive cells is significantly increased compared with that in the experimental group.

- CMC is a highly viscoelastic fluid; just like hyaluronan, which is widely accepted as a viscosupplement treatment for OA, it can also supplement and restore the viscoelasticity of joint fluid, thus improving the lubrication of the joint and alleviating the abnormal pressure on cartilage. As we know, aberrant mechanical force is a predisposing factor of $\mathrm{OA}$, and many researchers believe that the aberrant force on cartilage could translate into raised levels of MMPs. ${ }^{20}$ So alleviation of abnormal pressure on cartilage might mean less MMP-1 production.

- Many inflammatory cytokines participate in the development of OA. CMC, when given intra-articularly, could intercalate itself to the fissures and abrasions of cartilage and prevent the harmful cytokines from direct contact with the already damaged cartilage. Many of the cytokines such as interleukin 1 and NO increase the expression of MMPs. ${ }^{121}$

- The biodegraded product of CMC, glucosamine, could improve the mucopolysaccharide metabolism of cartilage-for example, by increasing the synthesis of aggrecan. This improvement would enhance the resistance of cartilage to OA causative factors. ${ }^{4}$

- Recent studies have shown that many chitinous materials could inhibit the synthesis of NO in in vitro cultured macrophages, suggesting that these materials could exert a direct effect on the cell biological function. ${ }^{1322}$ So, possibly, CMC could inhibit the synthesis of $\mathrm{NO}$ in mononuclear cells, which are increased in OA synovium, thus alleviating the inflammation of synovium and decreasing the production of MMPs.

Current treatments for OA are unsatisfactory, because few drugs have disease modifying ability. Our finding suggests that CMC could significantly reduce the severity of cartilage degradation and reduce the expression of MMP-1 in cartilage both at the mRNA and the protein level, We believe that CMC might modifying OA progression. The long term effects of CMC on OA and the need to determine its exact therapeutic mechanism suggest that further investigation is necessary.

\section{ACKNOWLEDGEMENTS}

We thank Dr Nagase $\mathrm{H}$ for his generous gift: the sheep antirabbit MMP-l serum.

\section{Authors' affiliations}

W Hongbin, D Jingyuan, Orthopaedic Department of Union Hospital, Tongji Medical College of Huazhong Science and Technology University, Wuhan 430022, People's Republic of China

C Linyun, D Yuming, Environmental Science Department, Wuhan University, Wuhan 430072, People's Republic of China

\section{REFERENCES}

1 Malemud CJ, Goldberg VM. Future directions for research and treatment of osteoarthritis. Front Biosci 1999:4:D762-71.
2 Huebner JL, Otterness IG, Freund EM, Caterson B, Kraus VB. Collagenase 1 and collagenase 3 expression in a guinea pig model of osteoarthritis. Arthritis Rheum 1998;41:877-90.

3 Klein R, Becker EW, Berg PA, Bernau A. Immunomodulatory properties of rumalon, a glycosaminoglycan peptide complex, in patients with osteoarthritis: activation of T helper cell type 2 cytokines and antigen-specific lgG4 antibodies. J Rheumatol 2000;27:448-54.

4 Deal CL, Moskowitz RW. Neutraceuticals as therapeutic agents in osteoarthritis. The role of glucosamine, chondroitin sulfate, and collagen hydrolysate. Rheum Dis Clin North Am 1999:25:379-95.

5 Takahashi K, Hashimoto S, Kubo T, Hirasawa Y, Lotz M, Amiel D. Effect of hyanuronan on chondrocyte apoptosis and nitric oxide production in experimentally induced osteoarthritis. J Rheumatol 2000;27:1713-20.

6 Sechriest VF, Miao YJ, Niyibizi C, Westerhausen-Larson A, Matthew HW, Evans $\mathrm{CH}$, et al. GAG-augmented polysaccharide hydrogel: a novel biocompatible and biodegradable material to support chondrogenesis. J Biomed Mater Res 2000;49:534-41.

7 Suh JK, Matthew HW. Application of chitosan-based polysaccharide biomaterials in cartilage tissue engineering: a review. Biomaterials 2000;21(24):2589-98.

8 Pelletier JP, Jovanovic D, Fernandes JC, Manning P, Connor JR, Currie MG, et al. Reduced progression of experimental osteoarthritis in vivo by selective inhibition of inducible nitric oxide synthase. Arthritis Rheum 1998;41:1275-86.

9 Mankin HJ, Dorfman H, Lippiello L, Zarins A. J Bone Joint Surg Am 1971:53:523-37.

10 Pelletier JP, Lascau-Coman V, Jovanovic D, Fernandes JC, Manning P, Connor JR, et al. Selective inhibition of inducible nitric oxide synthase in experimental oateoarthritis is associated with reduction in tissue levels of catabolic factors. J Rheumatol 1999;26:2002-14.

11 Han F, Ishiguro N, Ito T, Sakai T, Iwata H. Effects of sodium hyaluronate on experimental osteoarthritis in rabbit knee joints. Nagoya J Med Sci 1999;62:115-26.

12 Lu JX, Prudhommeaux F, Meunier A, Sedel L, Guillemin G. Effects of chitosan on rat knee cartilages. Biomaterials 1999:20:1937-44.

13 Hwang SM, Chen CY, Chen SS, Chen JC. Chitinous materials inhibit nitric oxide production by activated RAW 264.7 macrophages. Biochem Biophys Res Commun 2000;27:229-33.

14 Hashimoto S, Takahashi K, Amiel D, Coutts RD, Lotz M. Chondrocyte apoptosis and nitic oxide production during experimental induced osteoarthritis. Arthritis Rheum 1998;41:1266-74.

15 Muzzarelli RAA. Carboxymethylated chitins and chitosans. Carbohydrate Polymers 1988;8:1-21.

16 Brandt KD, Braunstein EM, Visco DM, O'Connor B, Heck D, Albrecht M. Anterior (cranial) cruciate ligament transection in the dog: a bona fide model of osteoarthritis, not merely of cartilage injury and repair. J Rheumato $1991 ; 18: 436-46$.

17 Tetlow LC, Adlam DJ, Woolley DE. Matrix metalloproteinase and proinflammatory cytokine production by chondrocytes of human osteoarthritic cartilage: associations with degenerative changes. Arthritis Rheum 2001:44:585-94

18 Ishiguro $\mathrm{N}$, Ito $\mathrm{T}$, Ito $\mathrm{H}$, Iwata $\mathrm{H}$, Jugessur $\mathrm{H}$, lonescu $\mathrm{M}$, et al. Relationship of matrix metalloproteinases and their inhibitors to cartilage proteoglycan and collagen turnover: analysis of synovial fluid from patients with osteoarthritis. Arthritis Rheum 1999:42:129-36.

19 Hollander AP, Pidoux I, Reiner A, Rorabeck C, Bourne R, Poole AR. Damage to type II in aging and osteoarthritis starts at the articular surface, originates round chondrocytes, and extends into the cartilage with progressive degeneration. J Clin Invest 1995:96:2859-69.

20 Jin G, Sah RL, Li YS, Shyy JY, Chien S. Biomechanical regulation of matrix metalloproteinase-9 in cultured chondrocytes. J Orthop Res 2000; 18:899-908

21 Pelletier JP, Faure MP, DiBattista JA, Wilhelm S, Visco D, Martel-Pelletier J. Coordinate synthesis of stromylisine, interleukin-1 and oncogene proteins in experimental osteoarthritis. An immunohistochemical study. Am J Pathol 1993; 142:95-105.

22 Lahiji A, Sohrabi A, Hungerford DS, Frondoza CG. Chitosan supports the expression of extracellular matrix proteins in human osteoblasts and chondrocytes. J Biomed Mater Res 2000;51:586-95. 